\title{
Re: "Ultrasound-Guided Block of the Axillary Nerve: A Prospective, Randomized, Single-Blind Study Comparing Interfascial and Perivascular Injections"
}

\section{TO THE EDITOR:}

We have read with great interest the recently published article about the interfascial shoulder blockade (1). In the aforementioned study, Dr. Kim and colleagues report that this modified approach is an easy, safe, and reproducible technique for regional anesthesia of the shoulder joint, particularly when compared with the perivascular approach originally proposed by Rothe et al (2). However, we would like to highlight some observations related to efficacy issues of the different techniques.

The posterior blockade of the axillary nerve (AN) described by both Rothe et al (2) and Kim et al (1) may miss articular branches due to the high percentage of anatomic variability (3). In fact, AN lie immediately in contact with the ventral surface of the subscapularis muscle, describing an oblique trajectory (from medial and cephalic to lateral and caudal) until the lower border of the lateral myotendinous insertion, where it winds dorsally crossing the quadrilateral space of Velpeau, and it divides in the anterior and posterior branches. At this level, the AN is in intimate relationship with the anterior and inferior part of the glenohumeral joint capsule, providing articular and capsular branches that may emerge completely from the AN before the quadrilateral space. Because of this anatomic issue, we have recently developed and published an anterior approach to the blockade of the AN, with the aim of blocking the articular branches of this nerve that until now could not be blocked with the approaches described earlier.

This might be achieved injecting, under ultrasound guidance, in the interfascial plane between the subscapularis and the deltoid muscles at the level of the subscapularis myotendinous junction (Fig. 1). * In accordance with Kim et al (1), we consider that an interfascial plane block offers safety and reproducibility. However, even when a fascial plane is the target, we still caution against intravascular injection, as the posterior circumflex humeral artery runs along the AN.

In our clinical experience and cadaveric study using this novel technique (3), we observed that the spread of the injectate also reaches the lateral pectoral nerve and the musculocutaneous nerve, thus complementing the anesthesia for shoulder interventions, in combination with suprascapular nerve block. This proposal is made in-line with the classical approach to regional anesthesia, in which different techniques are designed with the aim of individually blocking the nerves that provide sensory innervation to the target joint. However, a feasible alternative to the blockade of every possible articular branch is trying to block the terminal sensory branches in toto independently of their origin.

This may be achieved by injecting directly around (or even beneath) the capsular surface. Although this approach is not suitable for most joints, it is feasible to inject in the shoulder, in both the dorsal and the ventral surfaces of the myotendinous junction, reaching not only the surface of the shoulder capsule but also the intraarticular space. This is possible because the injectate may spread through a natural capsular gateway between the superior and middle glenohumeral ligaments, called the Weibrecht foramen (Fig. 1).

The possibility to target the AN and the articular space at the same time, the so-called shoulder anterior capsular block (SHAC block), opens the possibility of a rapid pain therapy in outpatient settings, relying on easily identifiable anatomic and ultrasonographic landmarks. In our opinion, this approach is easier to perform with the patient in a supine or beach chair position and may not need a prolonged learning curve for the physician.

Based on this clinical experience, we aim to perform clinical trials to confirm the role of the anterior blockage of the AN, alone or as SHAC block, and hereby call for interested participants to further this investigation. 


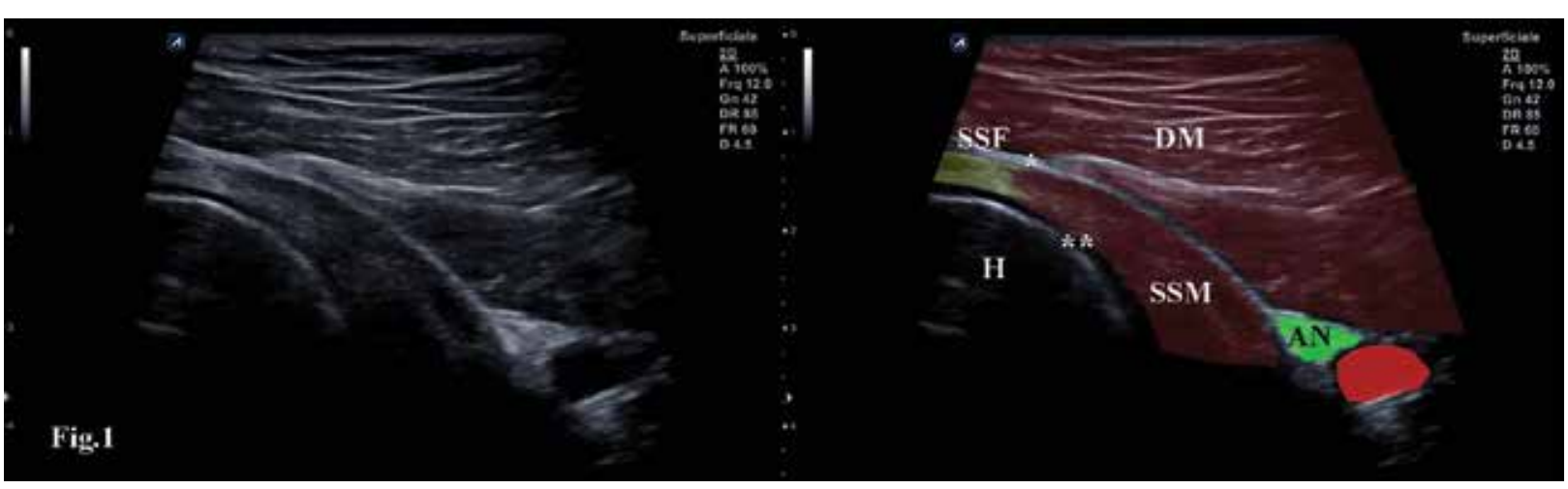

Fig. 1. Injectate spread through a natural capsular gateway between the superior and middle glenohumeral ligaments, called the Weibrecht foramen.

Felice Galluccio, MD

Department of Clinical and Experimental Medicine University of Florence AOU

Careggi, Italy

E-mail: felicegalluccio@gmail.com

Emilio Gonzalez Arnay, MD

Applied Anatomy Laboratory

Department of Anatomy, Histology, and

Neuroscience

Autonomous University of Madrid

Madrid, Spain

E-mail: emiliogonzalezarnay@gmail.com

Carlos Salazar, MD

Department of Anesthesia

Hospital Infanta Leonor

Madrid, Spain

E-mail: carhugos@gmail.com

Ece Yamak Altinpulluk, MD

Outcomes Research Department

Anesthesiology Institute, Cleveland Clinic

Cleveland, OH, USA

Department of Anesthesiology and Reanimation

Istanbul University-Cerrahpaşa

Cerrahpasa Medical Faculty

Istanbul, Turkey

E-mail: eceyamak@gmail.com
Marco Capassoni, MD

Department of Clinical and Experimental Medicine University of Florence AOU

Careggi, Italy

E-mail: marco.capassoni@gmail.com

Diego Simon Garcia, MD

Department of Anesthesiology and Reanimation Móstoles University Hospital

Madrid, Spain

E-mail: vk.diego@gmail.com

Karla Espinoza, MD

Hospital Mexico

San José, Costa Rica

E-mail: karlaespinoza08@gmail.com

Marilina Susana Olea, MD

Hospital Interzonal General Dr. José Penna

Bahia Blanca

Buenos Aires, Argentina

E-mail: oleamarilina84@gmail.com

Mario Fajardo Perez, MD

Department of Anesthesiology and Reanimation

Móstoles University Hospital

Madrid, Spain

E-mail: mfajardoperez@yahoo.es 


\section{References}

1. Kim ED, Baek JW, Kim JS, Oh SA, Kim $\mathrm{YH}$. Ultrasound-guided block of the axillary nerve: A prospective, randomized, single-blind study comparing interfascial and perivascular injections. Pain Physician 2019; 22:369-376.

2. Rothe $\mathrm{C}$, Asghar S, Andersen HL, Chris- 3 . tensen JK, Lange $\mathrm{KH}$. Ultrasound-guided block of the axillary nerve: A volunteer study of a new method. Acta Anaesthesiol Scand 2011; 55:565-570.
González-Arnay E, Jiménez-Sánchez L, García-Simón D, et al. Ultrasonography-guided anterior approach for axillary nerve blockade: An anatomical study. Clin Anat 2019 May 3. [Epub ahead of print]. 